\title{
Influence de la durée de l'exposition en jours courts sur la dormance des bourgeons de Gleditsia triacanthos L.
}

\author{
G.J. Aillaud ${ }^{1,2}$, A. Al Ibrahem ${ }^{1}$ et P. Neville ${ }^{1}$ \\ 1 Laboratoire de Morphogénèse Végétale (Aix-Marseille III), Faculté des Sciences et Techniques, \\ St-Jérôme, 13397 Marseille Cedex 13, et \\ 2 Laboratoire de Phytomorphologie Expérimentale (Université de Provence), Centre St-Charles, \\ 13331 Marseille Cedex 3, France
}

\section{Introduction}

Dans le cadre de nos travaux sur la dormance des bourgeons chez Gleditsia (Aillaud, 1982), et nous basant sur les travaux notamment de Nitsch $(1957,1957)$ et de Wareing (1949), nous avons étudié l'action de plusieurs durées d'exposition aux jours courts.

Neville (1969) a montré que la sénescence du bourgeon terminal n'était pas une condition suffisante pour qu'un plant soit considéré comme dormant (des bourgeons inactifs peuvent débourrer durant un certain temps après cette sénescence). Nous avons donc choisi des durées d'induction très diverses et certaines très longues.

\section{Matériel et Méthodes}

A partir de semis effectués à deux époques différentes (17 juillet et 7 novembre), des plants de Gleditsia triacanthos L. sont cultivés en salle de culture penclant un mois en photopériode "jours longs" (J.L., $16 \mathrm{~h}$ de jour). Le séjour en jours courts (J.C., $12 \mathrm{~h}$ de jour) est variable ( 16 traitements, donit la durée s'échelonne de 10 jours à 160 jours), avant retour en jours longs.

Observations et relevés: deux fois par semaine pendant plusieurs mois (état des bourgeons, hauteur des plants et nombre de feuilles étalées pour bien évaluer si la plante est en activité ou au repos. L'inertie au débourrement induite est calculée selon la formule de Harrington modifiée (Aillaud, 1982).

\section{Résultats}

tre série de tests: lots semés le 17 juillet

Le passage des plants en J.C. pendant 10,20 ou 30 jours entraîne un ralentissement progressif de la croissance et même un arrêt mornentané dans la dernière décade pour le séjour 30 jours en J.C. Mais dans tous les cas, la croissance reprend par le bourgeon terminal après le retour en J.L., et ce après un temps de latence plus ou moins long (Figs. $1 \mathrm{~A}$ et 2A). 

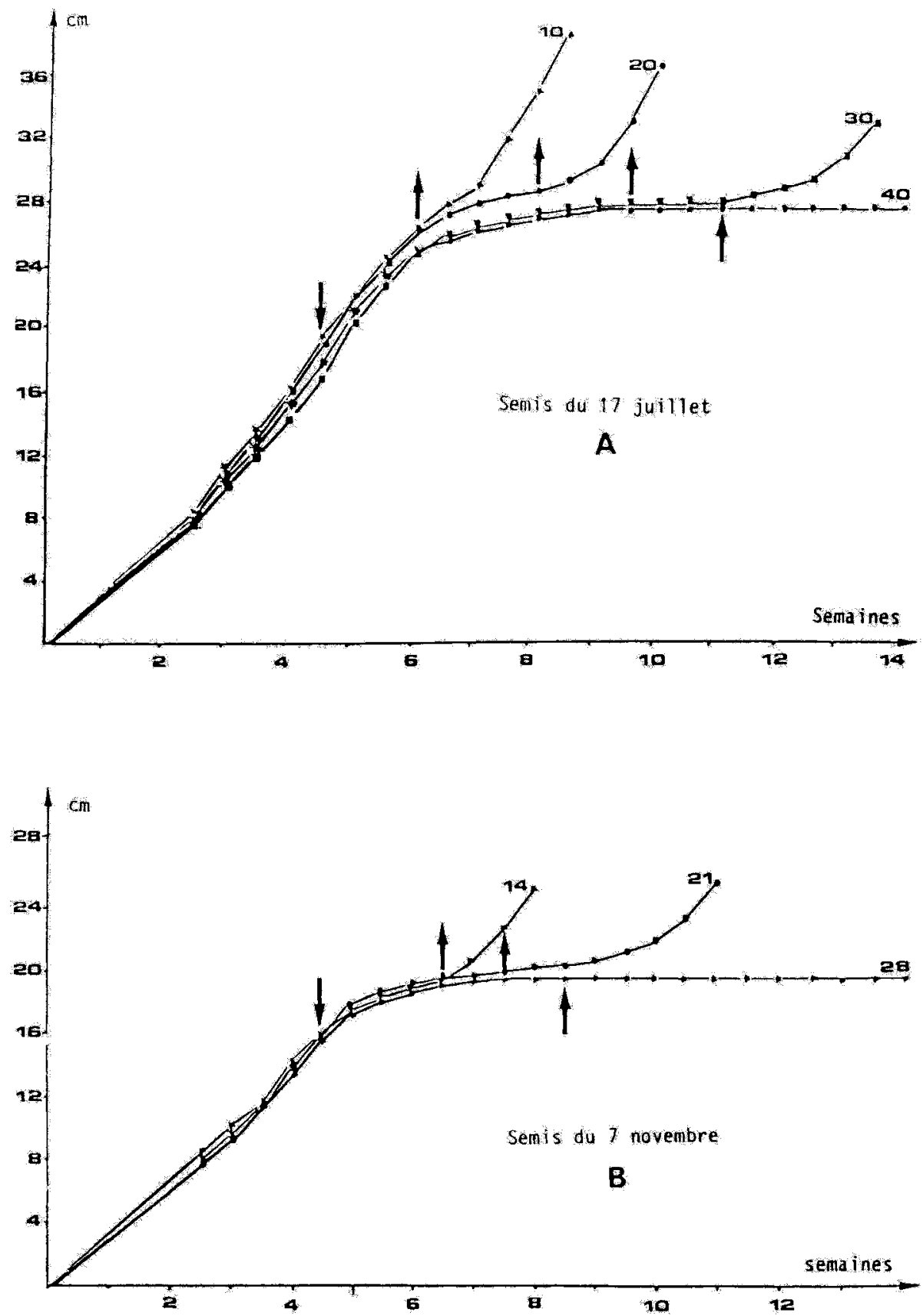

Fig. 1. Courbes de croissance de lots de plantes placés 1 mois en J.L. puis en J.C. ( $\downarrow$ ) pour : 10, 20,30 et 40 jours (A) ou pour : 14,21 et 28 jours (B) avant retour en $J L$. (T). Chaque point correspond a la moyenne pour les 10 plantes constituant chaque lot 

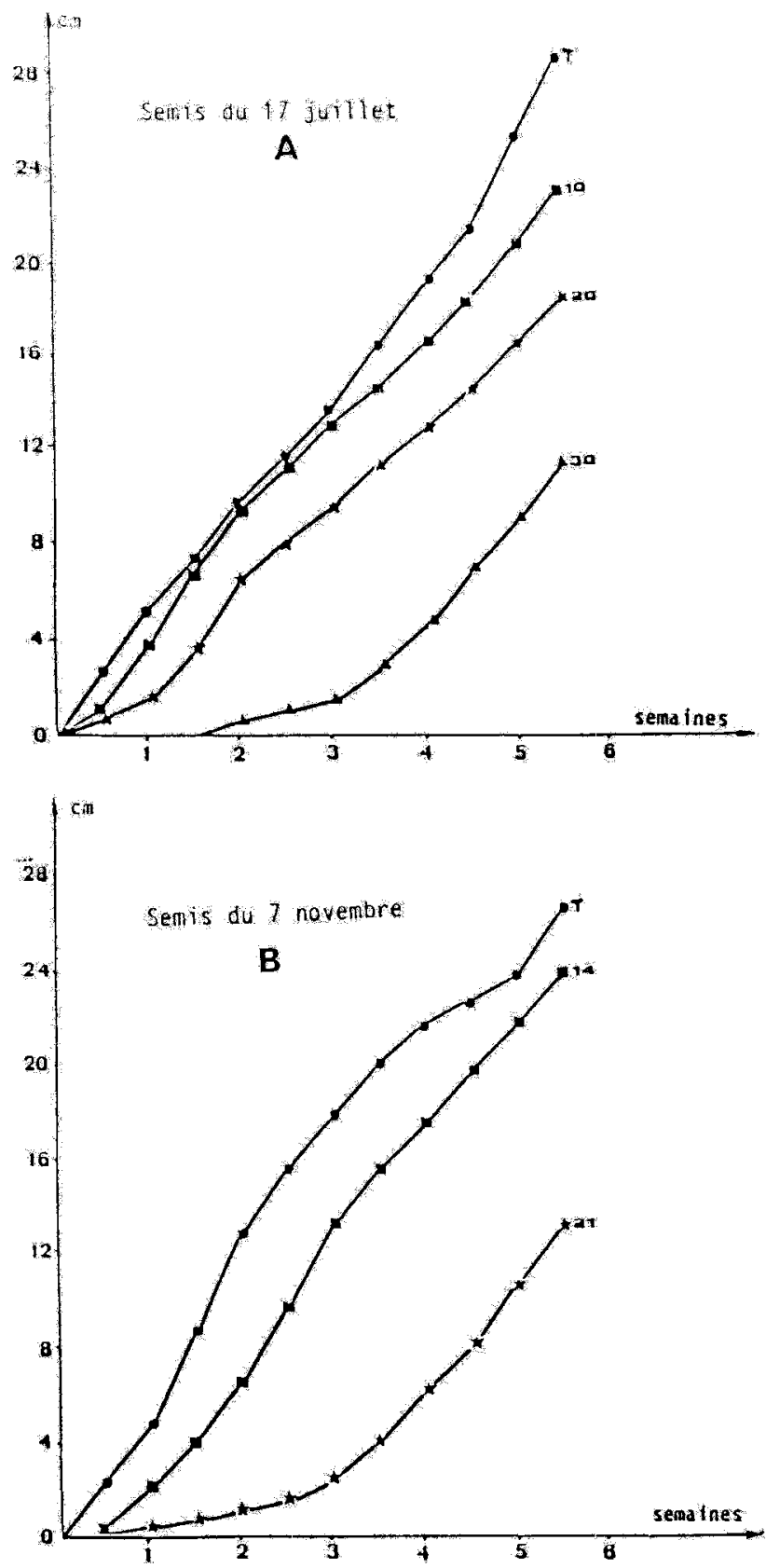

Fig. 2. Courbes de croissance après retour en JL. de lots de plantes ayant subi préalablement un sejour de $0(T=$ têmoins), 10,20 ou 30 jours (A) ou de $0(\mathrm{~T}=$ lémoins), 14 ou 21 jours (B) en JC. On constate que les plantes ne sont pas dormantes, mais que leur reprise de croissance est de plus en plus différée. Chaque point correspond à la moyenne pour les 10 plantes constituant chaque lot: 


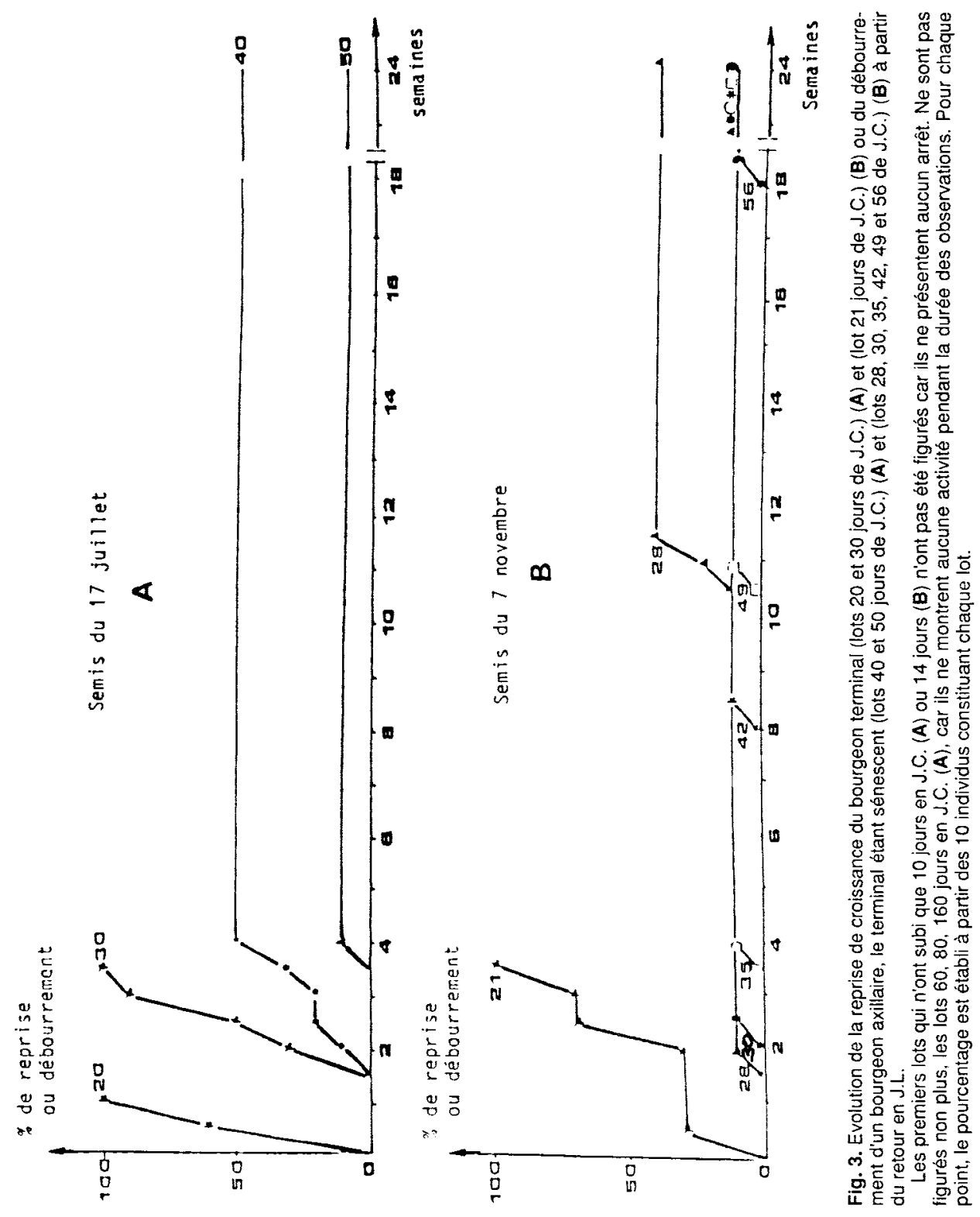


Les plantes qui séjournent de 40 à 160 jours en J.C. présentent un arrêt de croissance entre le $20^{\circ}$ et le $30^{\circ}$ jour, les bourgeons terminaux se nécrosent et tombent. La reprise a lieu (au niveau d'un bourgeon axillaire) pour une partie seulement des lots 40 et 50 jours en J.C.

Au-delà $(60,80$ et 160 jours en J.C.) toutes les plantes sont dormantes. L'inertie s'installe donc progressivement en fonction de la durée du séjour en J.C. Audelà de 30 jours, un nombre croissant de plantes deviennent dormantes et elles le sont toutes après 50 jours en J.C. (Fig. 3A).

$2^{e}$ série de tests: lots semés le 7 novembre

L'arrêt de la croissance, la perte du bourgeon terminal et l'installation de l'inertie sont plus rapides que pour les lots précédents: un séjour de 28 jours en J.C. provoque l'arrêt de croissance au bout de 25 jours, la perte des bourgeons terminaux et la dormance de 7 plantes sur 10 (Figs. 1B et 2B). Pour un séjour de 30 jours en J.C., on obtient $80 \%$ de plantes dormantes. Audelà de 35 jours, $90 \%$ du lot est dormant et le seul plant qui débourre le fait de plus en plus tard ( 126 jours pour le lot resté 56 jours en J.C.) (Fig. 3B).

\section{Discussion et Conclusion}

Comportement photopériodique et dormance du Gleditsia

Dans les conditions naturelles, après la germination, il y a étalement, outre les cotylédons de 13 à 15 feuilles, puis la croissance s'arrête, le bourgeon terminal devient sénescent et tombe (Neville, 1969; Aillaud, 1982). La dormance automnohivernale avec un maximum en octobre, est installée.
Expérimentalement, en conditions constantes de jours longs, les plantes sont capables d'avoir un développement accru. Les témoins poussent 18 à 20 semaines la croissance s'arrête, et, à partir de la 24 e semaine, le bourgeon terminal se nécrose et un bourgeon latéral donne une nouvelle pousse feuillée. Le transfert en J.C. ralentit ou stoppe la croissance en fonction de la durée. Le Gleditsia est donc une espèce à croissance végétative photopériodique dépendante.

Durée d'application des jours courts nécessaires pour induire la dormance avec trois stades dans l'inertie

En dessous d'un certain nombre de jours courts (14 à 30), le bourgeon terminal peut réduire ou arrêter son activité, mais le retour en jours longs permet la reprise. Dans un stade intermédiaire, le bourgeon terminal devient sénescent, (Neville, 1969) mais un certain nombre de bourgeons latéraux peuvent encore débourrer lors du retour en jours longs. Enfin, entre 35 et 50 jours de J.C., l'inertie basipète touche successivement tous les bourgeons, il y a induction de la dormance.

Importance dé la date d'application de ces jours courts

30 jours de J.C. suffisent pour induire à $90 \%$ la dormance d'un lot semé fin novembre alors qu'il en faut 50 pour atteindre le même résultat pour un lot semé début juillet. En dehors d'une variation d'un facteur autre que ceux contrôlés (température, humidité et durée du jours) il reste à envisager que notre espèce présente un changement d'état, qui la rende plus ou moins sensible à une induction de la dormance par les jours courts (que ce soit au niveau de la graine ou à celui de la 
plantule). Des expériences sont en cours pour vérifier ce point.

\section{Références}

Aillaud G.J. (1982) Etude de la dormance des bourgeons chez Gleditsia. I. Le cycle annuel de la jeune plante. Rev. Gén. Bot. 89, 97-109
Neville P. (1969) Morphogénèse chez Gleditsia triacanthos L. III. Etude histologique et expérimentale de la sénescence. Rev. Gén. Bot. 77, 353-385

Nitsch J.P. (1957) Growth responses of woody plants to photoperiodic stimuli. Proc. Am. Soc. Hortic. Sci. 70, 512-525

Nitsch J.P. (1957) Photoperiodism in woody plants. Proc. Am. Soc. Hortic. Sci. 70, 526-544

Wareing P.F. (1949) Photoperiodism in woody species. Forestry 22, 211-221 